\title{
Positioning, Structuring and Controlling with Nanoprecision
}

\author{
Regine Hedderich ${ }^{1,2}$, Tobias Heiler ${ }^{2,3}$, Roland Gröger ${ }^{2,3}$, \\ Thomas Schimmel ${ }^{2,3}$, and Stefan Walheim ${ }^{2,3}$ \\ ${ }^{1}$ Network NanoMat \\ ${ }^{2}$ Institute of Nanotechnology, Forschungszentrum Karlsruhe \\ Karlsruhe Institute of Technology (KIT), Northern Campus \\ 76344 Eggenstein Leopoldshafen, Germany \\ ${ }^{3}$ Institute of Applied Physics \\ and Center for Functional Nanostructures (CFN) \\ Universität Karlsruhe / Karlsruhe Institute of Technology (KIT), Southern Campus \\ 76131 Karlsruhe, Germany \\ regine.hedderichakit.edu \\ http://www. nanomat.de
}

\begin{abstract}
Key industries such as the automotive, electronic, medical and laboratory technical industries have continually rising demands for precise manufacturing, handling and control techniques. This is true for the manufacture of injection nozzles for engines as indeed also for the irradiation of extremely fine wafer structures and in the field of scanning probe microscopy. Some examples from research and their industrial application which have been made available by NanoMat Network Partners will be highlighted in this presentation. For example the spontaneous structure formation at the nanoscale and the application as anti-reflection layers.
\end{abstract}

Keywords: Nanotechnology, AFM, lithography, polymer blends.

\section{Applications}

The spontaneous structure formation in thin polymer films during spin coating can be used to define 2D and 3D chemically patterned surfaces, which feature complex and hierarchically organized structure motifs - both on the nanometer and on the micrometer scale. Investigations made by in situ light scattering during the typically five seconds of film formation give insight into the structure formation process [1]. An example of derivatives of these structures are bio-functionalizable structures with laterally defined spots of only $10 \mathrm{~nm}$ in diameter which are fabricated by the combination of polymer blend lithography and block copolymer nanolithography. By the use of micro-contact printing and scanning probe lithography - the self-organisation of the resulting polymer pattern can be controlled such that well ordered layoutdefined structures can be achieved [2]. Besides e-beam lithgography and printing 
techniques like micro-contact printing laser interference lithography can be used to generate appropriate Surface energy patterns on the substrates to guide the structure formation during spin-coating [3],[4]. But also a purely isotropic phase separation which take place on unstructured surfaces can lead to new physical properties. For instance, if we remove one of the two polymers of a morphology with a lateral length scale below $200 \mathrm{~nm}$, we are left with a nanoporous film. By tuning the composition of the polymer solution, the refractive index of our films can be adjusted in a range from 1.6 down to 1.05. This films can be used as high-performance anti-reflection coatings with outstanding optical properties [5].

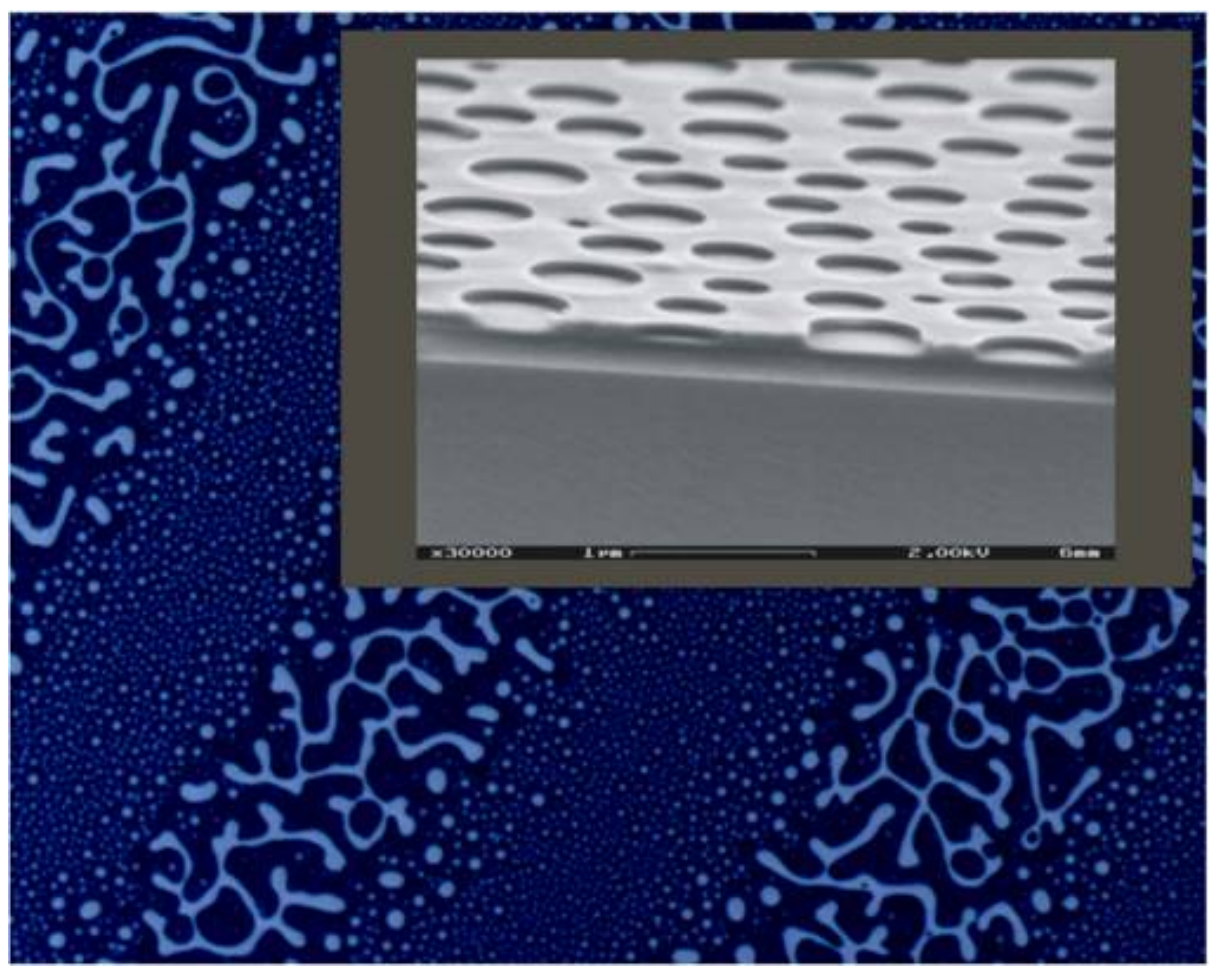

Fig. 1. Optical Microscopy image and SEM images of hierarchically structured surfaces made by polymer blend lithography. A complex structure formation process produces three characteristic correlation lengths at the same time. The diameter of holes in the upper picture is about $200 \mathrm{~nm}$ (scale bare: $1 \mu \mathrm{m}$ ). These polymer structures can be produced homogeneously on large samples and transferred into metal- and semiconductor structures.

Further researches in our Nanotechnology is controlled structuring of mica surfaces with the tip of an atomic force microscope by mechanically induced local etching. Here we show a reproducible structuring of surfaces of muscovite mica with the tip of 


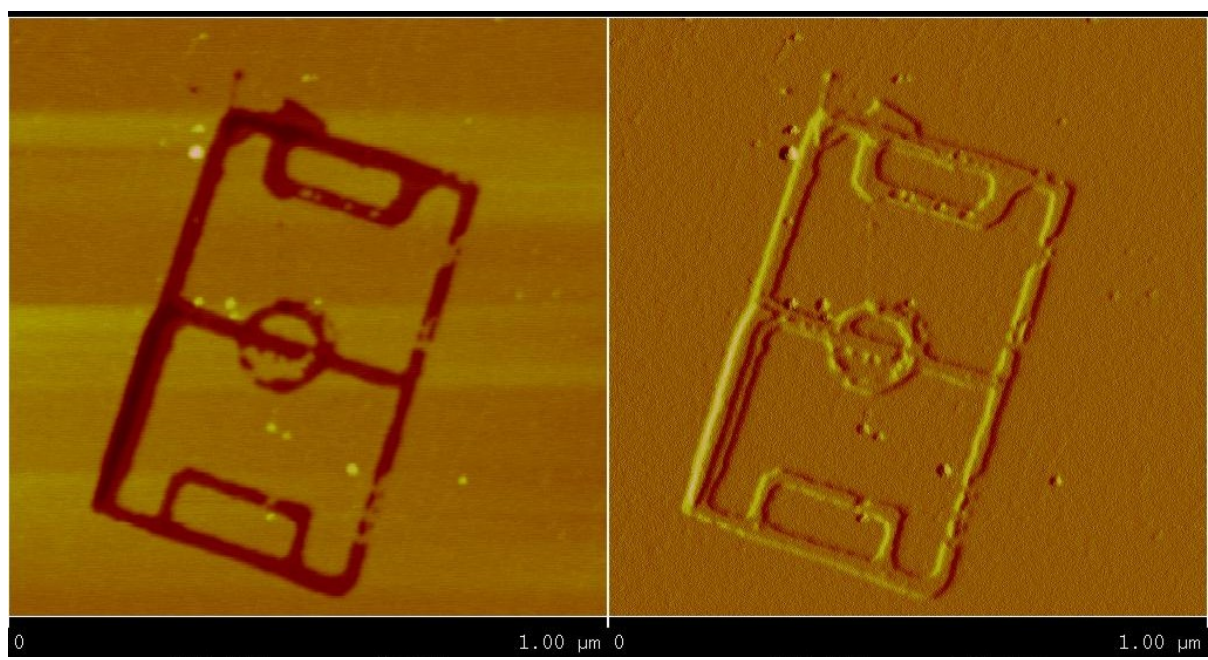

Fig. 2. Nanostructuring using our AFM-tip-induced mechanical etching process. A structure of $300 \mathrm{~nm} \times 700 \mathrm{~nm}$ in size was written on a mica surface. The groove width (FWHM) is $30 \mathrm{~nm}$ and the depth is about $3 \mathrm{~nm}$. The AFM images (image size $1 \mu \mathrm{m} \times 1 \mu \mathrm{m}$ ) of the resulting structures were taken in the constant amplitude tapping mode of the microscope: (left) topographic image: (right) amplitude deviation image.

an atomic force microscope under ambient conditions. By repeated scanning of the tip along a predefined pattern on a cleaved mica surface, mechanically induced etching can be observed on the atomic scale.

Line widths down to $3 \mathrm{~nm}$ were achieved, while at the same time patterns on a wide range of length scales between $5 \mathrm{~nm}$ and $100 \mu \mathrm{m}$ are generated reproducibly [6]. This can be explained by abrasive wear on the atomic scale due to sliding friction. The experiments allow the study of tribochemistry and abrasive wear on the atomic and molecular scale. At the same time, they represent an approach for high-precision structuring of surfaces within a wide range of length scales. The structuring of the topographic patterns was performed under ambient conditions. Neither debris particles nor ecrystallization of debris products on the surface was found. The former is an indication of the atomic-scale nature of the wear process. The latter is attributed to the fact that the experiments are performed under ambient conditions, including the presence of an adsorbed nanoscalewater layer. The experiments allow the study of abrasive wear under ambient conditions, at the same time providing a tool for the high-precision computer-controlled generation of patterns within a wide range of length scales from $1 \mu \mathrm{m}$ down to $3 \mathrm{~nm}$. By using highly linearized positioning components (3.5 $\mathrm{nm}$ repositioning accuracy / PI Karlsruhe, Germany) a range of up to $800 \times 800 \mu \mathrm{m}^{2}$ can be achieved. In a further combination of AFM structuring and a subsequent chemical reaction, protruding polymeric line patterns can be generated as a new way of constructive nanolithography [7]. 


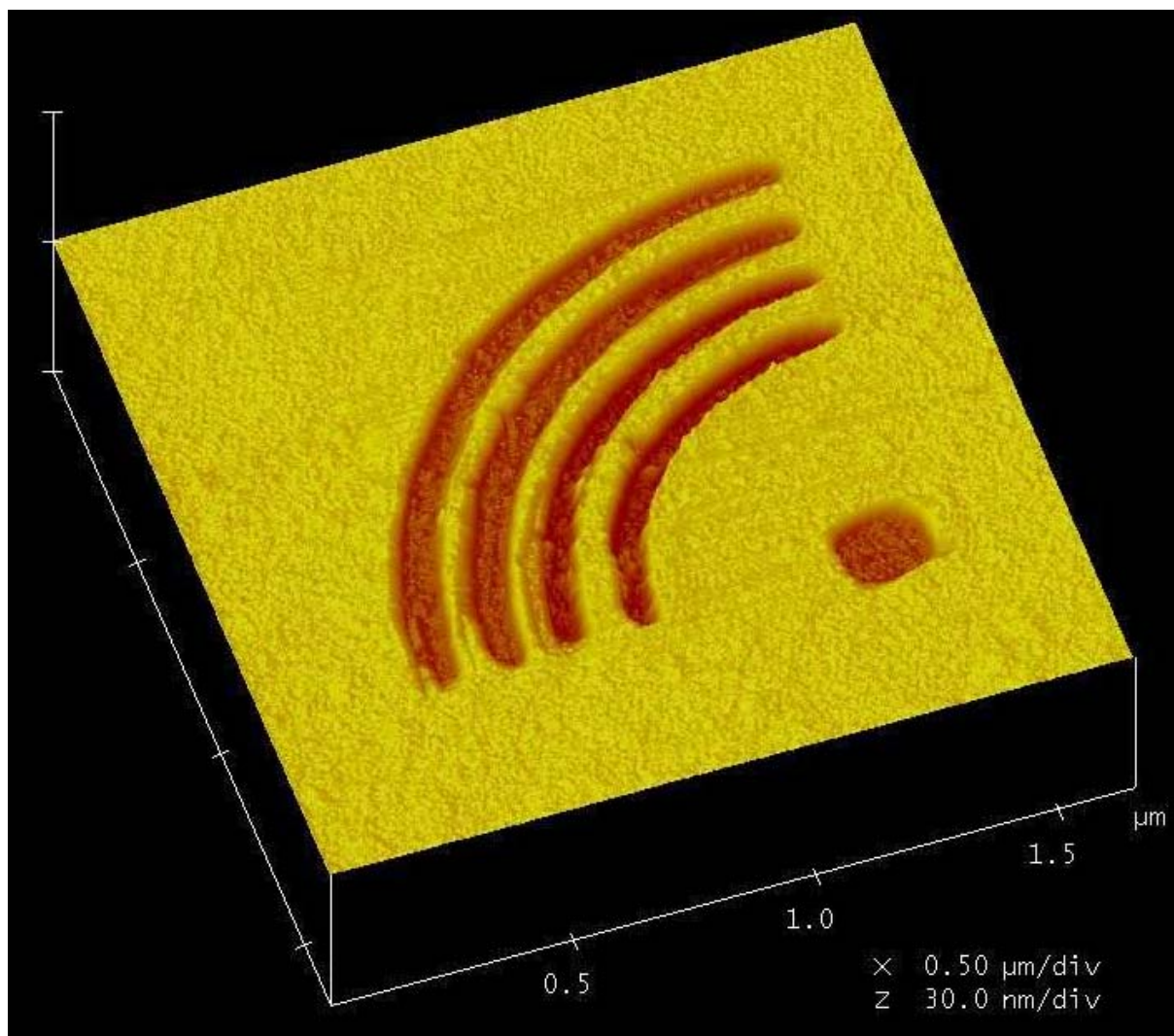

Fig. 3. Three dimensional image of another nano-machined structure in mica.(Depth: 3-5 nm)

Acknowledgments. We thank Patrik Dupeyrat for his experimental support.

\section{References}

1. Schmidt-Hansberg, B., Klein, M.F.G., Peters, K., Buss, F., Pfeifer, J., Walheim, S., Colsmann, A., Lemmer, U., Scharfer, P., Schabel, W.: In-situ monitoring the drying kinetics of knife coated polymer-fullerene films for organic solar cells. APL (2009) (accepted for publication)

2. Böltau, M., Walheim, S., Mlynek, J., Krausch, G., Steiner, U.: Surface-Induced structure formation of polymer blends on patterned substrates. Nature 391, 877 (1998)

3. Geldhauser, T., Boneberg, J., Leiderer, P., Walheim, S., Schimmel, T.: Generation of surface energy patterns by pulsed laser interference on self-assembled monolayers. Langmuir 24(22), 13155-13160 (2008)

4. Geldhauser, T., Walheim, S., Schimmel, T., Leiderer, P., Boneberg, J.: Influence of the Relative Humidity on the Demixing of Polymer Blends on Prepatterned Substrates Macromolecules (accepted, 2009), doi:10.1021/ma9022058 
5. Walheim, S., Schäffer, E., Mlynek, J., Steiner, U.: Nanophase-Separated Polymer Films as High-Performance Antireflection Coatings. Science 283, 520 (1999)

6. Müller, M., Fiedler, T., Gröger, R., Koch, T., Walheim, S., Obermair, C., Schimmel, T.: Controlled Structuring of Mica Surfaces with the Tip of an AFM by Mechanically Induced Local Etching. Surface and Interface Analysis 36, 189-192 (2004)

7. Barczewski, M., Walheim, S., Heiler, T., Błaszczyk, A., Mayor, M., Schimmel, T.: High Aspect Ratio Constructive Nanolithography with a Photo-Dimerizable Molecule. Langmuir (2009) (accepted for publication) 